\title{
Efectos en el examen final de la participación del estudiante en cuestionarios online
}

Ana Zorio-Grima ${ }^{a}$ y Paloma Merello-Gimenez ${ }^{b}$

${ }^{\mathrm{a}}$ Universidad de Valencia, ana.zorio@uv.es, ${ }^{\mathrm{b}}$ Universidad de Valencia, paloma.merello@uv.es ${ }^{\mathrm{b}}$

\begin{abstract}
This paper looks into the different evolution of students' online questionnaire performance and its impact on the final examination mark. This innovative technique has been used in a group of an introductory financial accounting course with 8 online questionnaires (one per unit). Using cluster analysis, we identify different groups of performance evolution. The evidence obtained suggests that in one of this groups a favourable test performance evolution may lead to overconfidence with the subsequent negative effect on the final examination mark. Future research with more variables and bigger samples will help identify this student profile with a view to preventing this undesired negative effect of this teaching technique.
\end{abstract}

Keywords:Moodle; accounting; online questionnaires

\begin{abstract}
Resumen
Este trabajo reflexiona sobre el distinto proceso de rendimiento de los estudiantes ante una innovadora técnica docente para sustentar la evaluación continua en un grupo de iniciación a la Contabilidad Financiera. Nuestro estudio se basa en el análisis cluster para identificar distintos tipos de perfil de los estudiantes según su rendimiento en 8 tests online autocorrectivos- uno por tema. Ello nos sugiere que en un determinado grupo de estudiantes una evolución favorable en los test puede conducir hacia un exceso de confianza de cara a la preparación del examen final y el subsiguiente efecto negativo sobre su nota en dicha prueba de sintesis. Futuros trabajos con mayor número de variables explicativas y muestra, podrán ayudarnos a identificar mejor a este perfil de estudiantes para tratar de prevenir este efecto no deseado del instrumento.
\end{abstract}

Palabras clave: Moodle; contabilidad; cuestionarios online

\section{Introducción}

En el nuevo Espacio Europeo de Educación Superior (EEES), el modelo educativo debe estar centrado en el estudiante (European Ministers of Education, 1999). Por ello, las Tecnologías de la Información y la Comunicación (TICs) resultan muy útiles en el proceso de enseñanza-aprendizaje (UNESCO, 2008) y han sido numerosas las experiencias de 
aplicación en distintas materias siendo sus resultados en general muy positivos (Swan, 2004; Garcia-Benau y Zorio-Grima, 2012).

Concretamente en el área de la administración y dirección de empresas, se ha estudiado recientemente una experiencia positiva sobre la aceptación de la plataforma MOODLE (Escobar-Rodriguez y Monge-Lozano, 2012). Algunos autores señalan el interés de la evaluación formativa, existiendo ya evidencia al respecto en el área concreta de la contabilidad (Einig, 2013).

Nuestras conclusiones alertan de un exceso de confianza entre los participantes en los test, especialmente entre determinados estudiantes.

Tras esta breve introducción, nuestro trabajo se estructura como sigue. En primer lugar describimos los objetivos de esta investigación. Luego presentamos la experiencia docente en sí, la muestra y metodología empleadas. La sección siguiente presenta los resultados. El artículo finaliza con un apartado de conclusiones.

\section{Objetivos}

Nuestro trabajo contribuye a la literatura en el sentido que identifica distintos tipos de estudiantes según su evolución en los tests y cómo alguno de estos tipos puede verse perjudicado por generar un exceso de confianza de cara al examen final, en línea con Gunn y McSporran (2003), sobre el dominio previo de la tecnología o el género.

Así pues, los objetivos de este trabajo consisten en describir esta experiencia docente, haciendo especial hincapié en la evolución del rendimiento de los estudiantes en los test online y su efecto sobre la nota alcanzada en el examen final.

\section{Desarrollo de la innovación}

Este mismo curso 2015/2016, se ha implementado la introducción de la plataforma Moodle en la Universidad de Valencia. Por ello, este es el primer año que se utiliza como herramienta docente y de evaluación en el curso de Contabilidad Financiera de primer curso del Grado en Administración y Dirección de Empresas de la Universidad de Valencia, impartida en el primer semestre.

La evaluación de esta asignatura se ha llevado a cabo por un examen final ( $80 \%$ nota final) y una evaluación continua (20\%), compuesta esta última por una prueba práctica en el aula (10\% nota final) y diferentes test teóricos $(10 \%)$.

En el curso académico 2015/2016 se han obtenido las siguientes calificaciones en primera convocatoria: un $26.42 \%$ de los alumnos ha obtenido una calificación de no presentado, un $33.96 \%$ ha suspendido (nota final $<5$ ), un $26.42 \%$ ha aprobado ( $5 \leq$ nota final $<7$ ) y un $13.21 \%$ ha obtenido una calificación de notable $(7 \leq$ nota final $<9)$. 
El temario consta de 9 unidades temáticas, de las cuales 8 han sido evaluadas a través de cuestionarios online autocorrectivos en la plataforma Moodle, combinando preguntas de respuesta múltiple y de verdadero/falso.

\subsection{Descripción de la muestra}

La asignatura se compone de 53 estudiantes, de los cuales un $32 \%$ son mujeres y un $68 \%$ hombres. El 81\% de los alumnos tienen una edad comprendida entre 18-24 años, un $9.43 \%$ entre 25-29, y el resto son mayores de 30 años. El 73.6\% es la primera vez que cursan la asignatura, un $15.1 \%$ la cursan por segunda vez y el $11.3 \%$ es al menos la $3^{\mathrm{a}}$ vez que la cursan.

Durante el curso 2015/2016, un $94.12 \%$ de las mujeres optaron por seguir el sistema de evaluación continua (16/17) frente a un $83.33 \%$ de los hombres (30/36). Del total de los 46 estudiantes que siguieron el método de evaluación continua, solo 39 se presentaron al examen final $(84.7 \%)$.

\subsection{Variables y Análisis de datos}

Se hace uso de la técnica de clasificación Análisis Clúster, en su modalidad jerárquica, cuyo principal propósito es agrupar objetos basándose en las características que poseen, intentando maximizar la homogeneidad de los objetos dentro de los conglomerados mientras que a la vez se maximiza la heterogeneidad entre los agregados (Hair et al, 1999).

Asimismo se implementa Análisis de Correspondencias Múltiples, un procedimiento para resumir la información contenida en una tabla de contingencia a través de un procedimiento objetivo de asignar valores numéricos a variables cualitativas (Peña, 2002).

Para el análisis de correspondencias múltiples, la nota media de los test y la nota del examen final se han categorizado en una variable de 4 niveles (suspendido, aprobado, notable, sobresaliente). Las equivalencias son suspendido $\mathrm{X}<5$ (variable=1), aprobado $5 \leq x<7$ (variable $=2)$, notable $7 \leq x<9($ variable $=3)$, sobresaliente $x \geq 9($ variable $=4)$.

\section{Resultados}

\subsection{Análisis de correlación de Pearson}

Se realiza un análisis de correlación de Pearson considerando como variables la nota del examen final y la nota de cada uno de los cuestionarios.

No se ha detectado ninguna correlación significativa entre la nota obtenida en el examen final y la nota obtenida en alguno de los 8 cuestionarios realizados.

Para el caso de los test, existe correlación significativa y de signo positivo entre los test del tema 3 y 4 ( $r=0.315$, sig=0.033), y los del tema 6 y 7 ( $r=0.339$, sig=0.047). Nótese que estos test hacen alusión a unidades temáticas consecutivas pero no específicamente relacionadas,

(c)) EY-NC-ND 2016, Universitat Politècnica de València

Congreso In-Red (2016) 
sea: "ciclo contable" (tema 3) y "Existencias" (tema 4), "Inmovilizado no financiero" (tema 6) y "Otros activos y pasivos financieros" (tema 7). Esto puede sugerir que las variables que condicionan los resultados obtenidos en el test no están únicamente relacionadas con la unidad temática a estudiar sino con otras variables como la cronología del curso, el calendario académico concreto de ese curso, la evolución de la motivación del alumnado a lo largo del curso, etc.

\subsection{Perfiles de alumnos en evaluación continua}

Se realiza un análisis cluster jerárquico sobre el alumnado, considerando como variables de clasificación las diferentes calificaciones obtenidas en cada uno de los cuestionarios.

Se escoge el método de Ward para minimizar las diferencias dentro del conglomerado y evitar problemas con el encadenamiento de la asignación. Entre las distintas alternativas, en cuanto a la medida de similitud entre los objetos, se ha optado por la distancia euclídea al cuadrado, dado que las variables se encuentran medidas en una escala común (0 a 10).

Como se ha desprendido del aparatado anterior, no existen problemas de multicolinealidad que condicionen los resultados del análisis.

Tras estudiar múltiples soluciones, se ha optado por seleccionar una solución con tres conglomerados.

Tabla 1. Numero de casos en la solución escogida.

\begin{tabular}{lll}
\hline Cluster & Casos & $\%$ \\
\hline 1 & 12 & $48 \%$ \\
2 & 4 & $16 \%$ \\
3 & 9 & $36 \%$ \\
Validos & 25 & \\
perdidos & 21 & \\
\hline
\end{tabular}

Tabla 2. Composición de los conglomerados

\begin{tabular}{lrrr}
\hline Variable & Cluster 1 & Cluster 2 & Cluster 3 \\
\hline T1 & 7,22 & 8,19 & 7,04 \\
T2 & 7,61 & 7,24 & 8,22 \\
T3 & 6,73 & 6,81 & 6,58 \\
T4 & 7,21 & N.P. & 8,06 \\
T5 & 7,52 & 7,25 & 7,87 \\
T6 & 6,75 & 7,00 & 7,41 \\
T7 & 7,17 & 6,50 & 8,11
\end{tabular}

(c)) EY-NC-ND 2016, Universitat Politècnica de València 


$\begin{array}{llll}\text { T8 } & 5,25 & 6,33 & 9,48\end{array}$

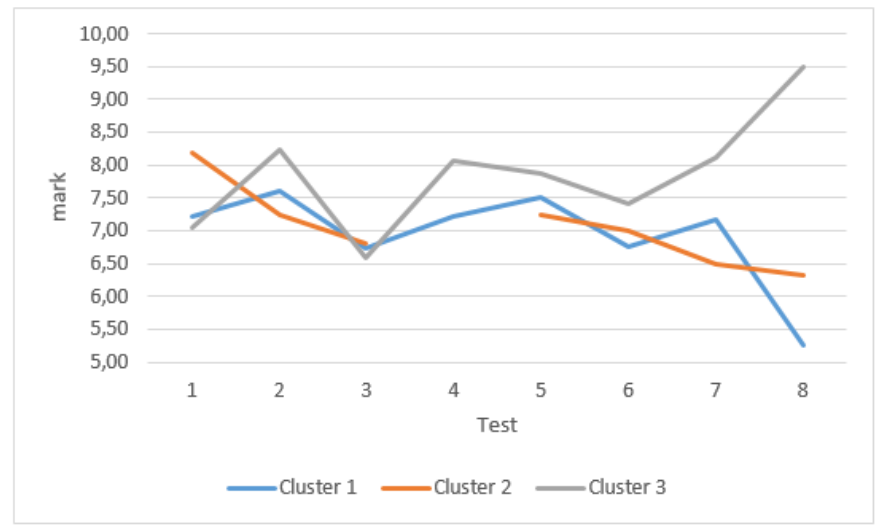

Figura 1. Evolución de la nota media por cluster.

De la figura 1 se desprende que los alumnos del Cluster 1 y 2 han seguido una evolución descendente en sus resultados de los cuestionarios de la evaluación continua, frente a los alumnos del cluster 3 que se caracterizan por una tendencia creciente en la nota obtenida en los test.

Asimismo, y para realizar una interpretación más visual de los diferentes conglomerados se ha realizado mediante un análisis de correspondencias múltiples la evaluación de las similitudes entre los diferentes perfiles de alumnos (según su resultado en los test) y la categorización de la nota media obtenida en los mismos. El resultado puede visualizarse en un mapa perceptual (Figura 2). El círculo azul muestra las 3 categorías de nota media en los test (1, suspenso; 2 ,aprobado 3 notable) y el círculo verde el cluster (1, 2 y 3 , ilustrados en las Tablas 1 y 2, así como en la Figura 1).

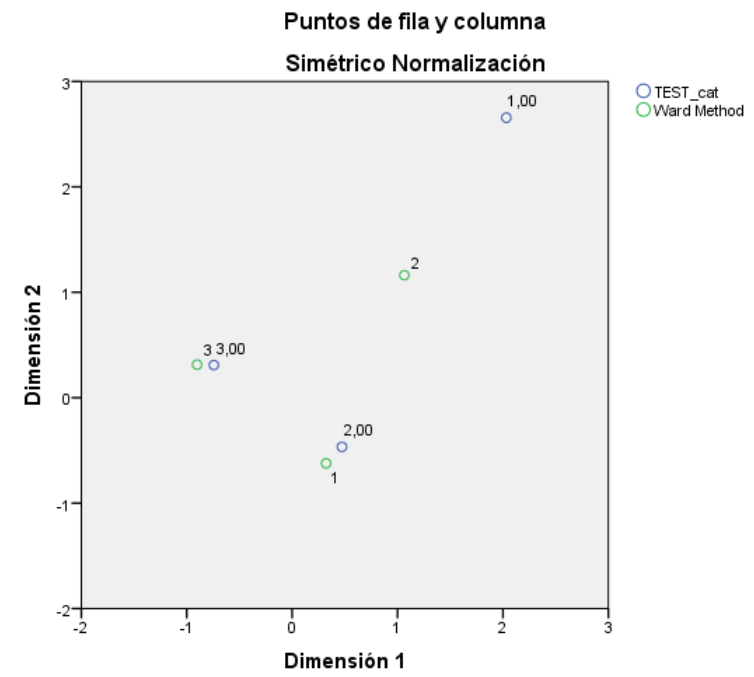

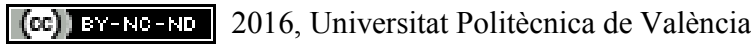


Figura 2. Mapa perceptual del análisis de correspondencias múltiples para la variable categórica Test y el resultado del análisis cluster.

Según la figura 2, y como era de esperar, aquellos alumnos que obtienen una nota media de notable en los test se corresponden principalmente con aquellos del cluster 3, los que no se presentan al test 4 (cluster 2) son los más cercanos a obtener una nota media de test de suspenso, y los del cluster 1 son aquellos que obtienen una nota media de aprobado.

\subsection{Valoración del impacto de la evaluación continua en Moodle sobre la nota del examen final}

En este apartado, mediante un análisis de correspondencias múltiples, se evalúan las similitudes entre los diferentes perfiles de alumnos (según su resultado en los test) y su calificación en el examen final. El mapa perceptual nos ayudará a definir el perfil de los alumnos que consiguen mejores resultados en la asignatura.

Se selecciona el número de dimensiones basándose en el nivel conjunto de explicación deseada de la variación y en el aumento de explicación ganado por la adición de una dimensión extra. Tómese como pauta incluir en el análisis aquellas dimensiones con inercia mayor que 0.2 , siempre teniendo en cuenta que una representación tridimensional, o menor, facilita la interpretación.

Así, ponderando el deseado aumento en la explicación con la interpretación, se juzga que una solución bidimensional es la más adecuada para el ulterior análisis.

Tabla 3. Dimensiones e inercias

\begin{tabular}{lcccr}
\hline & & & & \\
& & & & \\
Dimensión & Valor singular & Inercia & Proporción de inercia & Acumulado \\
\hline 1 & 0.249 & 0.062 & 0.789 & 0.789 \\
2 & 0.129 & 0.017 & 0.211 & 1.000 \\
Total & & 0.078 & 1.000 & 1.000 \\
\hline
\end{tabular}




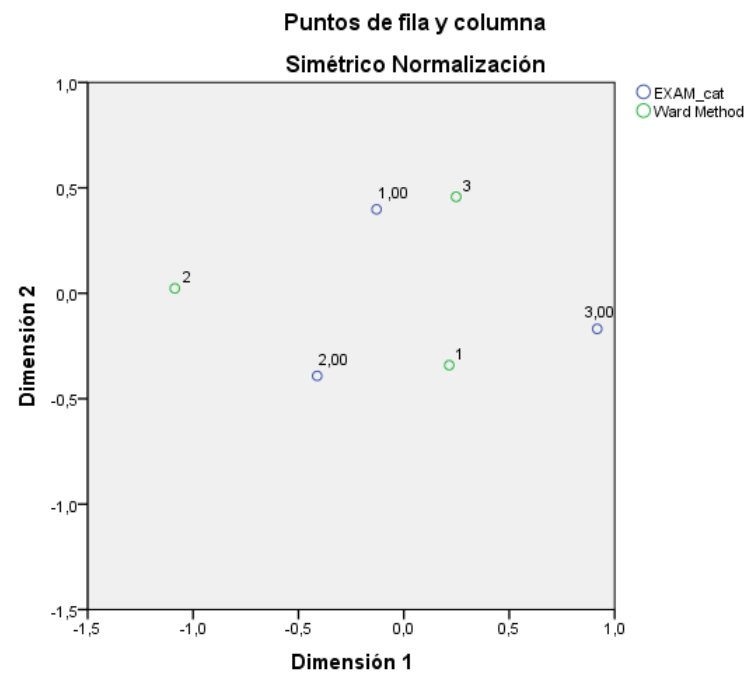

Figura 3. Mapa perceptual del análisis de correspondencias múltiples para la variable categórica Examen final y el resultado del análisis cluster.

De forma contraria a lo que quizá pudiera plantearse como hipótesis inicial, el mapa perceptual nos muestra que hay una relación entre aquellos alumnos que pertencen al cluster 3 y la categoría 1 de la nota de examen (suspenso). De otro lado, también hay una cierta similitud entre los alumnos pertenecientes al cluster 1 y la categoría 2 de la nota de examen (aprobado).

La categoría 3 de la nota de examen (notable) no se relaciona específicamente con ningún perfil de estudiante.

Este resultado puede estar indicando que existe una relación inversa entre la tendencia en los resultados obtenidos en la evaluación continua por un estudiante y la probabilidad de que supere el examen final. ¿Podría atribuirse esta relación a ciertos factores emocionales como un exceso de confianza? ¿La tendencia a un empeoramiento de la calificación de evaluación continua alienta al estudiante a realizar un mayor esfuerzo de estudio para el examen final?

\section{Conclusiones}

Resulta muy pertinente en el contexto de incorporación de las TICs a la evaluación formativa del alumnado, reflexionar sobre si realmente se logra con ello mejorar el rendimiento de forma eficiente. La literatura se hace eco de los distintos estilos de aprendizaje de los alumnos y cómo distintas metodologías pueden ser útiles para responder a esa variedad. Sin embargo en el área de la contabilidad los estudios previos no evidencian distintas pautas de evolución del nivel de conocimiento, ni su impacto en una prueba de síntesis final. Algunos trabajos señalan no obstante la posibilidad de que en ciertos contextos se dé un exceso de confianza basado en ciertas variables (como por ejemplo el dominio previo de la tecnología o el género), lo que nos lleva a plantear la necesidad de

\section{(c)) EY-NC-ND 2016, Universitat Politècnica de València}


seguir investigando el resultado de estas metodologías de enseñanza sobre muestras más grandes con una riqueza de variables que nos permita identificar los rasgos de los estudiantes con este perfil, para tratar de evitar estas consecuencias no deseadas del instrumento.

\section{Referencias}

EUROPEAN MINISTERS OF EDUCATION (1999). The Bologna Declaration of 19 June 1999 Joint declaration of the European Ministers of Education. Available at: http://www.ond.vlaanderen.be/hogeronderwijs/bologna/documents/MDC/BOLOGNA_DECLARATI ON1.pdf [Consulta: 28/01/2016]

EINIG, S. (2013). "Supporting Students' Learning: The Use of Formative Online Assessments". Accounting Education, 22(5), pp. 425-444.

ESCOBAR-RODRIGUEZ, T, y P. MONGE-LOZANO (2012). "The acceptance of Moodle technology by business administration students ”. Computers \& Education, 58 (4), pp.1085-1093.

GARCIA-BENAU, M. A., y A. ZORIO-GRIMA (2012). "Experience on the Use of a Mix of Teaching Methods in Higher Education of Accounting". Cuadernos de Contabilidad, 13(33), pp. 613657.

GUNN, C. y M. MCSPORRAN (2003). "Dominant or different? Gender issues in computer supported learning”. Journal of Asynchronous Learning Networks, 7, 1, pp.14-30.

HAIR, J. F. , R. E. ANDERSON, R. L. TATHAM, y W.C. BLACK, (1999). Análisis multivariante. Madrid, Spain: Prentice Hall Iberia.

PEÑA, D. (2002). Análisis de datos multivariantes. Madrid, Spain: McGraw-Hill.

SWAN, K (2004). "Learning online: A review of current research on issues of interface, teaching presence and learner characteristics" . Elements of quality online education: Into the mainstream, 5 , pp. 63-79.

UNESCO (2008). Estándares de competencias en TIC para docentes. Available at: http://www.oei.es/tic/UNESCOEstandaresDocentes.pdf. [Consulta: 27/01/2016] 